\title{
Effect of Electrode Biasing on the Radial Electric Field Structure Bifurcation in Tokamak Plasmas
}

\author{
N. Kasuya 1), K. Itoh 2), Y. Takase 3) \\ 1) Graduate School of Science, University of Tokyo, Tokyo, Japan \\ 2) National Institute for Fusion Science, Toki, Japan \\ 3) Graduate School of Frontier Sciences, University of Tokyo, Tokyo, Japan \\ e-mail contact of main author: kasuya@ plasma.phys.s.u-tokyo.ac.jp
}

\begin{abstract}
The mechanism for formation of a steep structure in the radial electric field is a key issue in plasma confinement. Properties of the radial electric field bifurcation are studied taking into account the effect of electrode biasing. The radial electric field structure is determined by the charge conservation equation. From the nonlinear mechanism associated with local current due to ion bulk viscosity, a transition can take place. Various types of radial electric field structures with multiple peaks are allowed for the same boundary condition. The ion orbit loss term breaks the symmetry of the radial current similarly to the ambipolar radial electric field. A radial current driven by the electrode plays the role of a control parameter in a transition similarly to the pressure gradient. A phase diagram is given in the spontaneous drive vs. external drive space. Differences in the radial shape of solitary electric field structures are demonstrated in the presence of spatial varying components. This study clarifies the mechanisms of nonlinear structure formation in transport barriers.
\end{abstract}

\section{Introduction}

Many experiments have shown the importance of the radial electric field in improved confinement states [1,2] and several theories can explain the transition mechanism $[3,4]$. The mechanism for formation of a steep structure in the radial electric field is a key issue in plasma confinement. In TEXTOR, H-mode was induced by electrode biasing in the plasma edge region, and the spatial profile of the radial electric field changed suddenly from a flat one to a peaked one [5]. In this paper properties of the radial electric field transition between L-mode and H-mode are studied taking into account the effect of electrode biasing. The analysis gives various kinds of solutions, and a possibility to attain wider and stronger transport barriers in fusion devices is discussed.

\section{Model Equation}

The radial electric field structure is determined by the charge conservation equation $[6]$,

$$
\frac{\partial}{\partial t} E_{\mathrm{r}}=-\frac{1}{\varepsilon_{0} \varepsilon_{\perp}}\left(J_{\mathrm{r}}+J_{\mathrm{visc}}-J_{\mathrm{ext}}+J_{\mathrm{oth}}\right)
$$

where $J_{\mathrm{r}}$ is the local current due to ion bulk viscosity [7], $J_{\text {visc }}$ is the current driven by shear viscosity [8], $J_{\text {ext }}$ is the current driven into the electrode by the external circuit, $J_{\text {oth }}$ represents other contributions, such as orbit losses [3,4] and charge exchange losses with neutrals [9], $\varepsilon_{0}$ is the vacuum susceptibility, and $\varepsilon_{\perp}$ is the dielectric constant of a magnetized plasma. In a 
stationary state, the normalized form of the model equation can be written as

$$
\frac{\partial^{2}}{\partial x^{2}} X-\left(X-X_{\mathrm{a}}\right) f(X, y)-g(X, y)+I=0,
$$

where $X=E_{\mathrm{r}} /\left(\mathrm{v}_{\mathrm{ti}} B_{\theta}\right), \quad y=r v_{\mathrm{ii}} B /\left(\mathrm{v}_{\mathrm{ti}} B_{\theta}\right), \quad I=J_{\mathrm{ext}} /\left(\mathrm{v}_{\mathrm{ti}} B_{\theta} \sigma(0)\right), \quad f(X, y)=\operatorname{Im} Z(X, y), \quad x=\left(r-r_{0}\right) / l$, and $l=\sqrt{\mu_{\mathrm{i}} \varepsilon_{0} \varepsilon_{\perp} / \sigma(0)}$. Hear $X_{\mathrm{a}}$ is the ambipolar radial electric field, which gives zero radial current, $Z(X, y)$ is the plasma dispersion function, $\mathrm{v}_{\mathrm{ti}}\left(\equiv \sqrt{2 T_{\mathrm{i}} / m_{\mathrm{i}}}\right)$ is the ion thermal velocity, $v_{\mathrm{ii}}$ is the ion collision frequency, $\mu_{\mathrm{i}}$ is the shear viscosity of ions, $\sigma(0)$ is the conductivity for zero radial electric field and $B_{\theta}$ is the poloidal magnetic filed. In the definition above, $X$ is the normalized radial electric field, $y$ is the normalized collision frequency, $I$ is the normalized external current and $x$ is the minor radius normalized by $l$, which represents the magnitude of the viscosity. The radius $r_{0}$ is chosen to be the mid-point between the electrode and the limiter. The function $f(X, y)$ is the conductivity in an axisymmetric confined plasma, given by the neoclassical transport process. The function $g(X, y)$ comes from $J_{\text {oth }}$ and in this paper ion orbit losses are considered. The first term of Eq. (2) comes from $J_{\text {visc }}$ and acts as a diffusion term due to anomalous shear viscosity. The ambipolar radial electric field depends on the gradient of each plasma parameter. The profiles of plasma parameters, such as the ion density and the ion temperature, contribute to the radial current through this term. We use this term as the control parameter that describes the plasma state.

\section{Solutions of the Equation}

The nonlinear mechanism associated with $J_{\mathrm{r}}$ is studied first, setting $J_{\mathrm{oth}}=0$ [10]. Various types of radial electric field structure with multiple peaks are allowed for the same boundary condition $\partial X / \partial x=0$. Figure 1 shows examples of the spatial profile of the radial electric field: flat ones (X1 and X2), and peaked ones with single, double and triple peaks (a, $\mathrm{b}$ and $\mathrm{c}$ ), respectively, are illustrated. The solutions with multiple peaks imply states in which the transport barrier has a wider radial extent. This shows the possibility of further confinement improvement. The relationship between the applied voltage $V_{\text {ext }}$ and the electrode current $I$ is obtained. The circuit includes a resistance. Figure 2 shows $V-I$ curves for the case with $d=18, y=0.1$ and $\hat{r}=4.0$ where $d$ is half the distance between the electrode and the limiter, and $\hat{r}$ is the resistance of the external circuit normalized by $\sqrt{\mu_{\mathrm{i}} \varepsilon_{0} \varepsilon_{\perp} / \sigma(0)^{3}}$. Spatially constant solutions exist on branches T1 and T2. S1 and S2 are solutions having solitary structures with one and two peaks, respectively. Both stable and unstable regions exist in stationary solutions and their boundary points are the critical points for transition from one state to another. Point B in Fig. 2 is where a transition takes place. A stability analysis shows that branch $\mathrm{S} 1$ is taken after the transition, though there exist two available states with the same applied voltage [11]. For application to realistic cases, the ambipolar radial electric field is included. A phase diagram is given in the spontaneous drive (e.g., pressure gradient) vs. external drive (i.e., external bias voltage) space with various control parameters (e.g., collisionality). The analysis gives a threshold value of $500 \mathrm{~V}$ for the applied voltage and $80 \mathrm{~A}$ for the electrode current for plasma parameters of the TEXTOR experiment. These values agree with the experimental results. A change in the radial electric field affects the viscosity [6]. Including this effect is straightforward and does not affect the conclusion. 


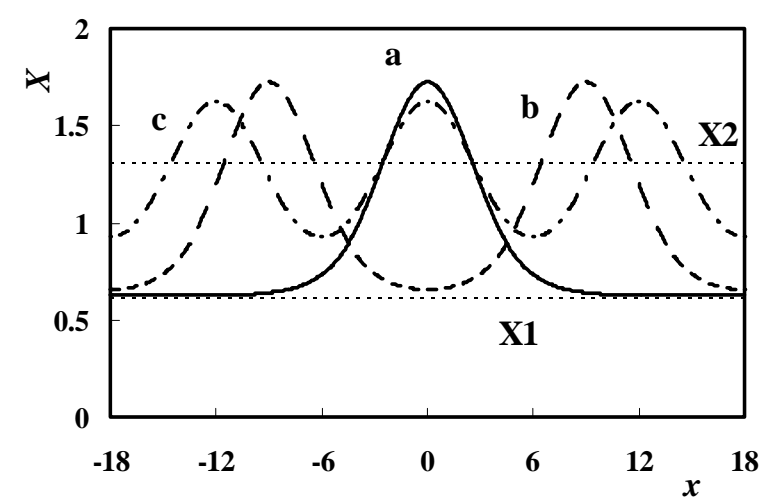

FIG. 1. Solitary structures of the radial electric field for $d=18, y=0.1$ and $I=0.5 . x$ is $a$ spatial coordinate and $x=0$ corresponds to the mid-point between the electrode and the limiter.

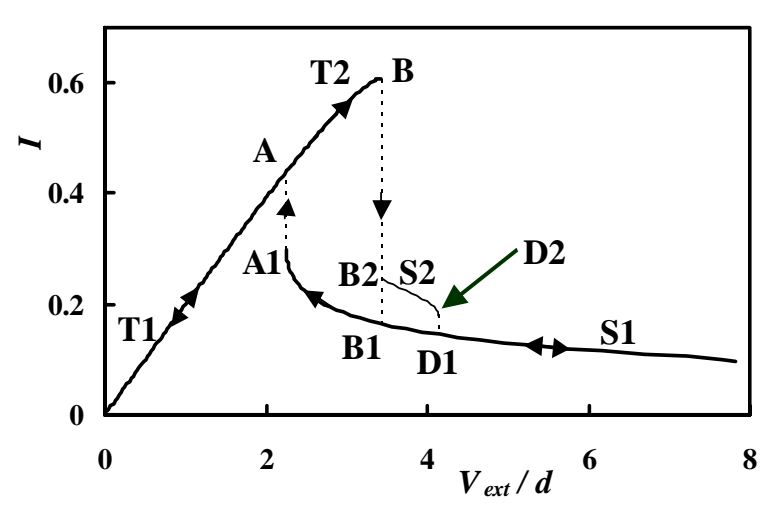

FIG. 2. The relationship between the applied voltage and the current when $d=18, y=0.1$ and $\hat{r}=4.0$.

\section{Comparison of the Spontaneous and Forced Transitions}

We next studied the effect of electrode biasing on spontaneous transition induced by the ion orbit loss [3]. The model of ion orbit loss in Eq. (2) is based on Ref. [4]. Two nonlinear terms affect the radial electric field in this case. The ion orbit loss term breaks the symmetry of the radial current similarly to the ambipolar radial electric field that depends on the pressure gradient. As the pressure gradient increases, a spontaneous hard transition takes place when the collision frequency is small and squeezing of the banana orbit is strong. The radial electric field is negative for the case with $I=0$. When a current is driven by the electrode, it plays the role of a control parameter in a transition similarly to the pressure gradient. The threshold for transition by electrode biasing changes in accordance with the pressure gradient.

\section{Asymmetry in Polarity of the Applied Voltage}

There is an asymmetry in polarity of the applied voltage [5]. It comes from the competition between two nonlinear terms. For the positive biasing case, various solitary solutions can exist, and a radial electric field transition can take place even though the profiles of plasma parameters become steeper. On the contrary for the negative biasing case, the two nonlinear terms have opposite signs, so the sum of the nonlinear terms can have more than one maxima or minima and the number of spatially constant solutions increases in such a condition. A radial electric field transition does not take place when the profiles of plasma parameters become steeper. Only a soft transition can take place. Figure 3 shows the phase diagram of the radial electric field structure for $d=20$ in the $X_{\mathrm{a}}-V_{\text {ext }}$ space. The threshold voltage for transition is given by the boundaries of the shaded regions. The boundaries neighboring $\mathrm{S}$ and $\mathrm{T}$ give the forward transition threshold and the backward transition threshold, respectively. In Fig. 3 only solutions that has one peak are considered. When the distance between the electrode and the limiter is large, the competition between the two nonlinear terms can give a larger number of solutions. 


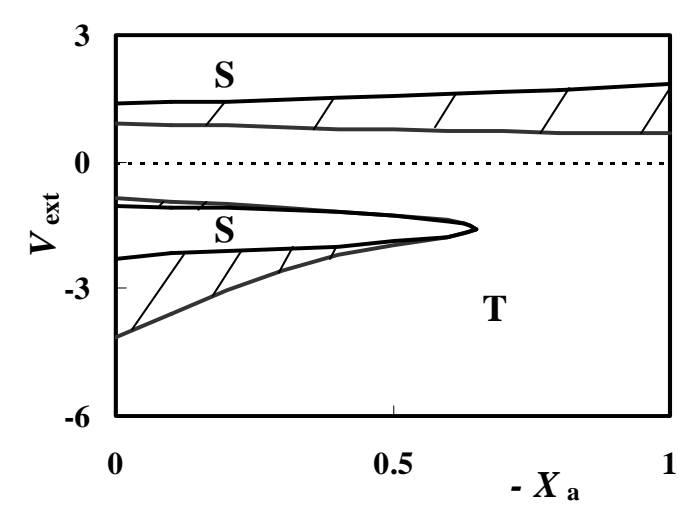

FIG. 3. Phase diagram of the radial electric field structure for $d=20$. $S$ and $T$ represent regions where a solitary solution and a spatially constant solution exist, respectively. The shaded regions indicate where both solutions can exist and gives hysteresis.

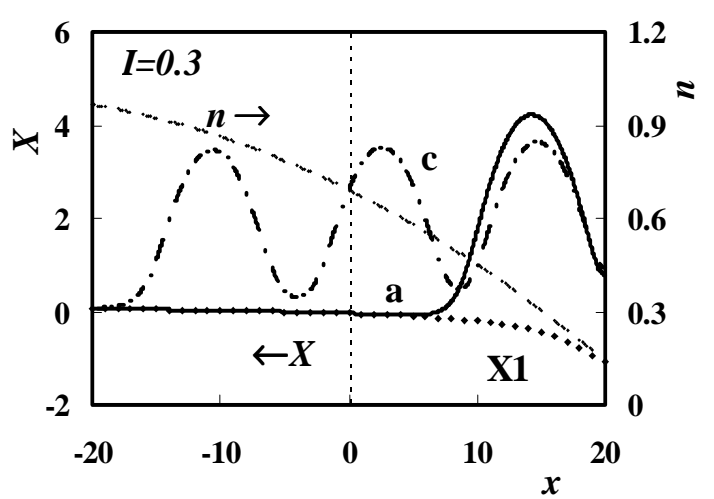

FIG. 4. Effect of a non-uniform background density profile (dashed line) for the case with $I=0.3$ and $d=20$.

\section{Spatial Asymmetry of the radial electric field structure}

We assume the ambipolar radial electric field term to be constant in space, so Eq. (2) has translation invariance in space. In reality there exist spatial variations in plasma parameters, and their non-uniformities affect the structure of the radial electric field through the ambipolar radial electric field. In addition the magnitude of the orbit loss term depends on the spatial position and also affects the shape of the solitary radial electric field. Here we consider these two factors to be possible causes of spatially asymmetric structures. These terms make the nonlinear response terms in Eq. (2) vary in space, and these spatial variations give other scale lengths. Figure 4 shows spatial profiles of the radial electric field with a non-uniform background ion density. A large density gradient has a drawing effect. Peaks of the radial electric field shift to the larger density gradient region for positive biasing. Radial electric field structures with single and triple peaks are shown in Fig. 4. The peaks approach the outermost peak position of the structure that has the largest number of peaks (the triple peaks structure in Fig. 4). The shape of the structure is not sensitive to the asymmetric term but only a small non-uniformity gives a significant change in the peak position. The structures must satisfy the boundary conditions at the electrode and the limiter, and they restrict the change of the structure. The peak settles where the drawing and restricting effects balance. A peak that exists far away from the boundaries can easily gain a large shift, so the first peak of each structure converged to the same position as is shown in Fig. 4. The selection rule for transition [11] shows that the structure with one peak is formed after a transition, but this sensitivity of the peak position to spatial gradients indicates that the peak of the one-peak structure appears at the peak position of the structure with the maximum number of peaks. The maximum number of available peaks is determined by the distance between the electrode and the limiter, so a measurement of the peak position gives information on the scale of the normalizing distance $l$. The spatial variation of the orbit loss term has the same tendency to change the nonlinear response term in Eq. (2), so it also affects the peak position of the radial 
electric field in the same way. Peaks of the radial electric field are shifted outward in electrode biasing experiments [5]. These non-uniformity effects are possible causes of spatially asymmetric structures that have been observed in experiments. The non-uniformity makes discontinuity in the relationship between the voltage and the electrode current. This effect appears near the $\mathrm{L} / \mathrm{H}$ transition points.

\section{Conclusions}

We have analyzed the radial electric field bifurcation including the effect of electrode biasing in tokamak plasmas. This work is an extension of the previous H-mode theory [3,4], so spontaneous transition is included as a special case when $I=0$. We considered neoclassical bulk viscosity, ion orbit losses, anomalous transport and electrode current to be the dominant contributors to the radial current, and the nonlinear responses of the former two terms to the radial electric field give various structures with multiple peaks for the same boundary condition. The competition between two nonlinear terms gives a unique feature. Their balancing explains the $\mathrm{H}$-mode transition mechanism and an asymmetric bifurcation feature with respect to the electrode current term. Components that give spatial asymmetry are also considered. Only a small spatial variation can cause a large shift of the peak position, so that a measurement of the peak position gives important information on the scale length. The radial electric field contributes to improving plasma confinement. Solitary structures with multiple peaks derived here can give wide large-gradient regions. Such structures are stable, so once they are formed, they can exist stably. A double peak structure can be obtained by inserting one more electrode at the mid-point of the biasing region and improve plasma confinement further. Electrode biasing can thus be used to make wider and stronger transport barriers.

\section{Acknowledgements}

It is a pleasure to thank Prof. S.-I. Itoh, Prof. A. Fukuyama and Prof. M. Yagi for useful discussions. This work is partially supported by the LHD Project Research Collaboration Programme and by the Grant-in-Aid for Scientific Research of MEXT Japan.

[1] BURREL, K. H., Phys. Plasmas 4 (1997) 1499.

[2] IDA, K., Plasma Phys. Control. Fusion 40 (1998) 1429.

[3] ITOH, S.-I., ITOH, K., Phys. Rev. Lett. 60 (1988) 2276.

[4] SHAING, K. C., CRUME, E. C., Jr., Phys. Rev. Lett. 63 (1989) 2369.

[5] WEYNANTS, R. R., et al., Nucl. Fusion 32 (1992) 837.

[6] ITOH, K., ITOH, S.-I., YAGI, M., FUKUYAMA, A., Phys. Plasmas 5 (1998) 4121.

[7] STRINGER, T. E., Nucl. Fusion 33 (1993) 1249.

[8] ITOH, K., ITOH, S.-I., FUKUYAMA, A., Transport and Structural Formation in Plasmas, IOPP, Bristol (1999).

[9] CARRERAS, B. A., DIAMOND, P. H., VETOULIS, G., Phys. Plasmas 3 (1996) 4106.

[10] KASUYA, N., ITOH, K., TAKASE, Y., Plasma Phys. Control. Fusion 44 (2002) A287.

[11] KASUYA, N., ITOH, K., TAKASE, Y., Bifurcation Phenomena in Plasmas (ITOH, S.-I., KAWAI, Y., Ed.), Kyushu University Press, Fukuoka (2002) 341-354. 